\title{
Correction of machining operations with the Total Inertial Steering
}

\author{
A. Boukar*, M. Pillet, E. Pairel, and A. Sergent \\ SYMME Laboratory, University of Savoie, BP 80439, 74944 Annecy-le-Vieux Cedex, France
}

Received: 20 October 2013 / Accepted: 28 April 2014

\begin{abstract}
The Total Inertial Steering approach proposed in this paper can perform an optimum correction of the geometric deviations of the manufactured part with respect to its digital model, from the measured points on its surfaces. In the case of production by machine tool numerical control, there exist a link between the tool offsets and deviations of measured points. An incidence matrix which represents this link is obtained. In most cases, this matrix is not square and therefore not invertible, because there are more measured points as correctors to adjust. The Gauss pseudo-inverse is used to calculate the values of corrections to be made to compensate for measured deviations. Tolerances associated with the surfaces must also be taken into account in the incidence matrix. However, when the same cutting tool machine two surfaces with different point values, the resulting solution favors the one with the highest number of points, at the expense of the other surface which can remain not conform towards its tolerance. This paper proposes a strategy to rebalance the correction surfaces, and this regardless of the number of points and tolerance of each surfaces. A relatively simple tutorial example is given in the paper to enable tracking calculations.
\end{abstract}

Keywords: Machining; machine setting; corrector; inertial steering; balancing surfaces

\section{Introduction}

The Total Inertial Steering approach (TIS) is presented in this article builds on previous work made by Denimal et al. [1] and Pillet [2] on inertial steering. It is improving his work in providing an effective solution to calculate the corrections taking into account the number of points of the surfaces. The principle of TIS approach is to minimize the inertia, i.e. the average of squared deviations between the target and the measured points along the normal to the surface. When a part is measured in output production, there are always deviations between the finished part and its digital model. To bring closer the machined part to this model, the machining dispersions must be master by acting on the correctors available on machine. This article is not concerned to calculate or to estimate the machining dispersions. The reader is invited to refer for example to experimental and simulation work by Tichadou et al. [3] and Sergent et al. [4] on identification and evaluation of machining defects.

However, this paper proposes to determine the optimal values of corrections to be made in the machine to reduce the geometric deviations. About this topic, several authors have worked to try to close as possible the finished product to its original model defined by design. Zilong and Del Castillo [5] proposed a method of successive adjustment

\footnotetext{
^ Correspondence: boukar.abdelhakim@univ-savoie.fr
}

of the machine using a dynamic programming formulation based on a Bayesian estimation of unknown variance which minimizes the quadratic off-target costs. They showed by starting with unknown variance at the start of production, that an optimum setting of the machine is obtained when this variance is estimated from samples of the product. Del Castillo et al. [6] and Rong [7] have developed a sequential process adjustment based on a stochastic approximation to approximate the average value to the target. They used the rule of Grubbs [8] which is to adjust the machine by a successive approach. Martin et al. [9] showed that the geometrical adjustment of manufacturing defects could be done using the principle of the small displacements torsor. The authors Pairel et al. [10,11] and Goldschmidt [12] proposed a method called Copilot-Pro ${ }^{\circledR}$ which adjusts all cutting tools for finishing and roughing. The method is to adjust the machine by measuring the most relevant manufacturing dimensions, determined from the plan of process adjustment.

Pillet and Pairel [13], and Pillet et al. [14] proposed to introduce an adjustment rule in the algorithms of the inertial steering, by calculating the most probable offsetting taking into account the natural dispersion and the measured deviation of the manufacturing process. This solution is to weight the measured deviation by a factor between 0 and 1 which depends on the short term dispersion, the sample size and the measured deviation itself. The idea is that the correction should be proportional 
to the ratio deviation/random dispersion; this avoids the threshold effects of Shewhart control charts.

Based on this work, this paper complete the TIS approach by introducing a rule that balances the setting surfaces machined by the same cutting tool when the number of points probed is not identical. A simple theoretical example is given in this paper for ease of explanation.

\section{The total inertial steering}

The objective of any production is to produce parts conforms to the requirements of the geometry established by CAD (Computer Aided Design). This requirement is materialized by a digital target on which is added an acceptable level of variability (tolerances). The Total Inertial Steering (TIS) approach is a tool that is able to reconcile the real workpiece to its digital model through the measured points on all surfaces of the workpiece. Inertia is the quality indicator of the part in inertial steering. The inertia of a surface is calculated by equation (1) [2]:

$$
I=\sqrt{\frac{\sum_{i=1}^{n}(e i)^{2}}{n}}
$$

$e i$ : difference between the actual point and the target point, measured along the local vector normal to the surface;

$n$ : Number of points measured on the surface.

The principle of the TIS approach is to establish a direct relationship between the setting parameters available on the machine (i.e. tool offsets) and the points of the surfaces expressed in a frame of reference linked to the machine. It directly uses all raw data available (the deviation on each measured point). There is "no loss of information" induced by passing through a classic parameterization of the geometry such as lengths, diameters, angles, ... This advantage provides a level of setting accuracy far more than conventional approaches.

Figure 1 gives a geometrical specification of the finished part. The notch, in green, has a tolerance lower than the elliptical pocket in yellow.

A block of rectangular material with dimensions $25 \mathrm{~mm} \times 20 \mathrm{~mm}$ is machined on a CNC milling machine. It is fixed to the milling machine table. Three stops are used to position the block on the table of the milling machine. A clamping system ensures its fixation (see Fig. 2). An elliptical pocket is realized in the block by contour milling and a notch by diving of the same tool (toric milling cutter).

\subsection{Construction of the inertial reference frame}

For doing inertial steering, the points and the normals must expressed in the Machine frame reference, i.e., more precisely, in the axes of displacement of the tools. Indeed it is along these axes that correctors operate. Initially the axes of the program reference frame are parallels to the axes of the machine reference frame. The program reference frame is the axis system in which is expressed the

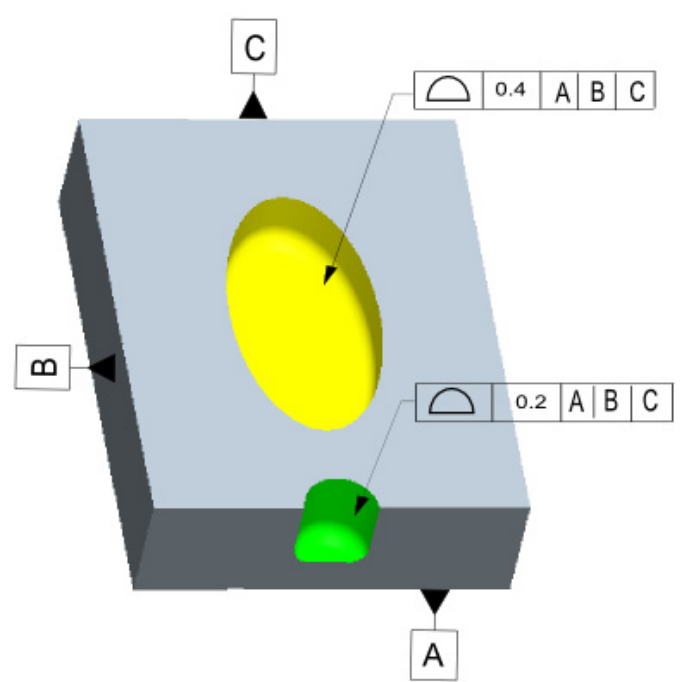

Fig. 1. Inertial specification of the part.

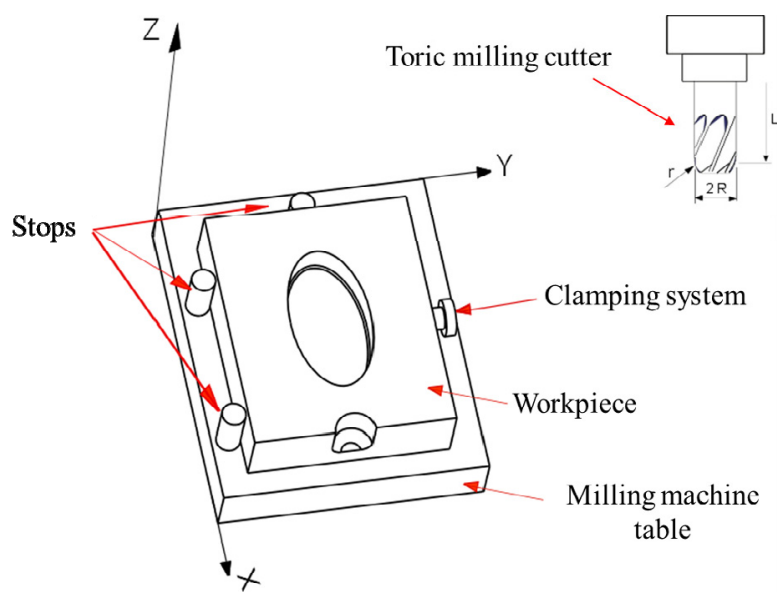

Fig. 2. Assembly workpiece machining.

crossing points of the NC program and wherein the shapes are machined (in blue on Figs. 3 and 4). The workpiece reference frame is built from the datum system and is used for measuring the elliptical pocket and the notch wherein the theoretical position of the elliptical pocket and the notch are known (in dotted red on Figs. 3 and 4). The workpiece reference frame is constructed by measuring on surfaces A, B and C of the workpiece inside machine tool or outside the machine.

The Program reference frame is positioned by probing of a point on surfaces of the block supported on stops (see Fig. 2). However, because of misalignment of the stops with regard to the machine axes, this Program reference frame does not correspond perfectly to the workpiece reference frame. Otherwise, the initial value of the tool radius offset and tool length offset of the cutting tool are not perfects so that the size and the depth of the pocket and the notch are not correct. The total inertial steering will enable to correct all these deviations in a single step of calculation starting from the measured deviations on a set of probed points on machined surfaces. Thus the program reference frame is moved to better correspond to the 


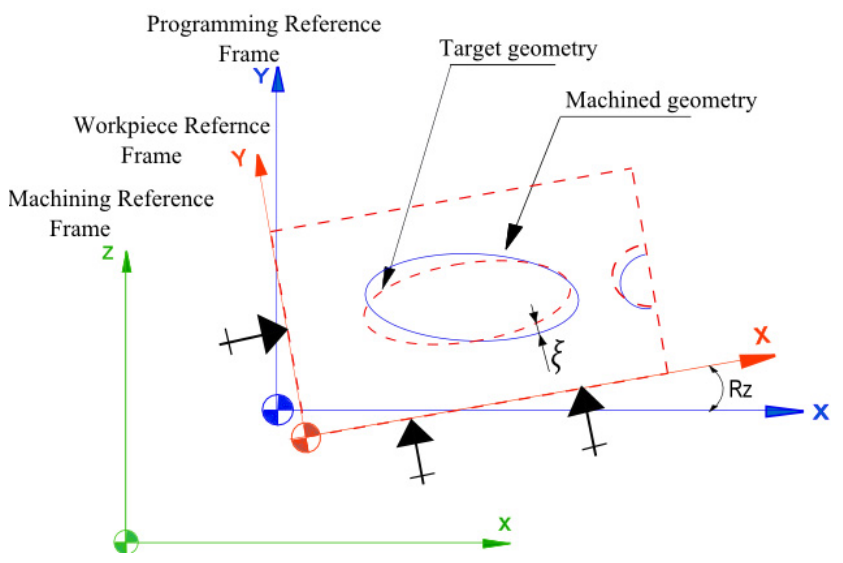

Fig. 3. Initial position of shapes machined.

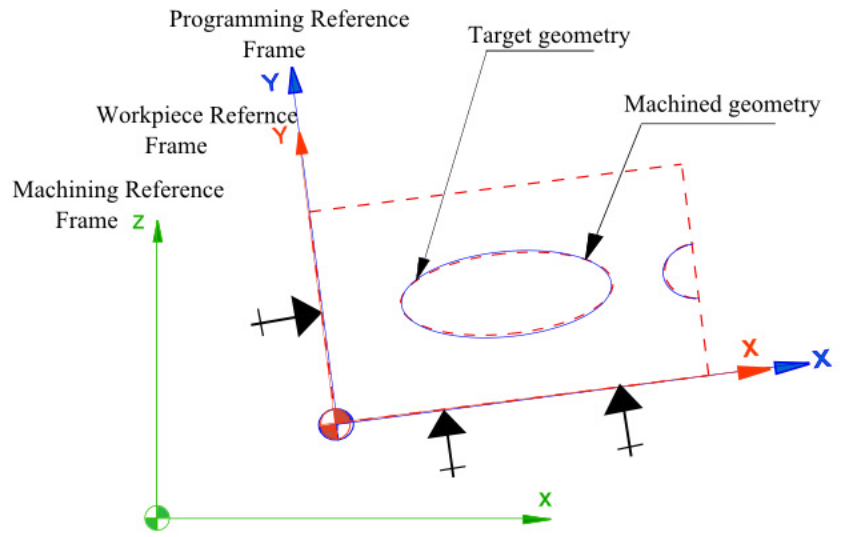

Fig. 4. Final position of shapes machined.

workpiece reference frame (Fig. 4) and tool offsets will be corrected. Figures 3 and 4 give a 2D representation respectively the initial and final position of the elliptical pocket and notch before and after correction.

\section{Construction of the incidence matrix [a] of the correctors on machined surfaces}

\subsection{Choice of the points}

Points are arbitrarily measured on the workpiece. Eleven points are measured on the elliptical pocket S1 (eight points on its side S1.1, three on the bottom S1.2) and three points on the notch $\mathrm{S} 2$ to adjust the tool. The points on the notch are measured on its cylindrical portion to allow repositioning the notch relative to the ellipse. Surfaces of the fillets radiuses generated by the end milling radius and surfaces of the bottom of the notch are not probed. If the fillets radiuses are not the good shape, the tool will regrind or replace. But in the case of this paper, the fillets radiuses are not corrected. Figure 5 shows a representation of points probed in the workpiece frame reference. The points (P1, P2 .. P8) of S1.1 are precisely measured for each $45^{\circ}$ angle relative to the axes of the ellipse in the center of the ellipse.

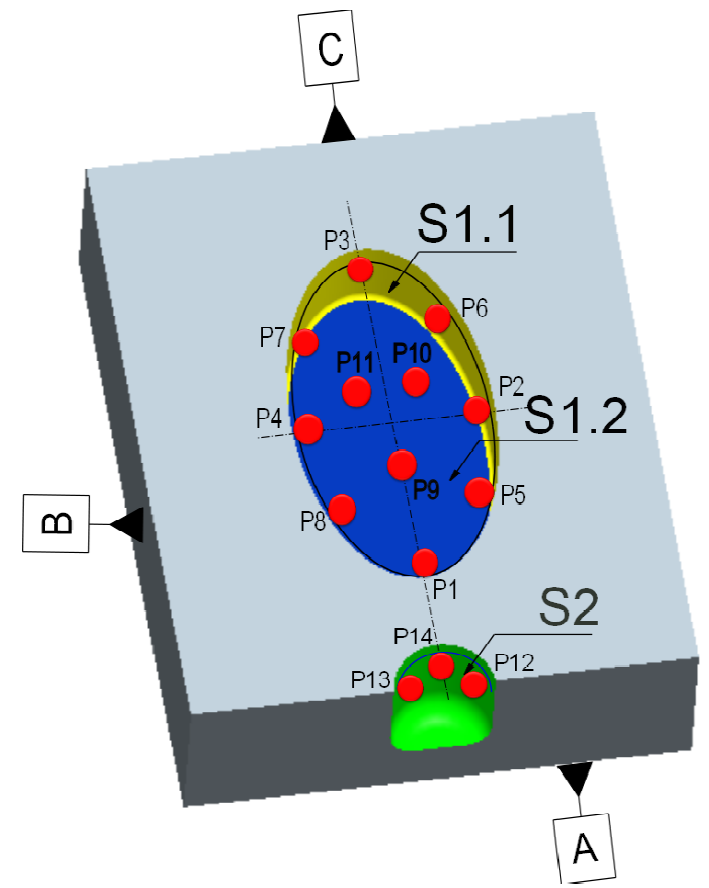

Fig. 5. Representation of measured points in the workpiece reference frame.

Table 1 gives the coordinates of points and normals expressed in the workpiece reference frame, and the deviations of these points along the normal surfaces. Residual deviations after correction are given in the Appendix.

\subsection{Method for calculating incidence matrix and steering matrix}

Surfaces S1.1, S1.2 and S2 are generated by the same cutting tool. This tool can be adjusted by acting on the tool length offset $(L)$ along the $Z$ axis and on its tool radius offset $(R)$. Further, the displacement variables $T x, T y$, and $R z$ are introduced in the program to enable repositioning the program reference frame on workpiece reference frame to refocus the machined shapes on their targets (see Sect. 2.1). Correctors $T x, T y$ and $R z$ have the effect of moving the machined surfaces while $R$ has the effect of changing their "sizes". $R z$ is a rotation about the $Z$ axis of the program reference frame. The deviation between the target surfaces and machined surfaces is measured on several points by distances along the normal to the surfaces at these points. Displacements and variations are small in size, then it is possible to linearize these distances relative to these components using the method of small displacements (Bourdet and Clement [15]) defined by equation (2):

$$
e i=\xi i+a i T x+b i T y+c i L+N i R z+\varphi i R,
$$

with:

$\xi i$ : initial deviation relative to its target position; $e i$ : final deviation after correction; $a i, b i, c i$ : direction cosines of the local normal to the target surface; 
Table 1. Points expressed in the workpiece reference frame.

\begin{tabular}{|c|c|c|c|c|c|c|c|c|c|}
\hline Surface & Tolerance & Point & $X$ & $Y$ & $Z$ & $a i$ & $b i$ & $c i$ & $\xi i$ \\
\hline \multirow{8}{*}{ S1.1 } & \multirow{8}{*}{0.4} & P1 & 20 & 10 & 5 & -1 & 0 & 0 & 0.077 \\
\hline & & P2 & 12.5 & 14 & 5 & 0 & -1 & 0 & 0.090 \\
\hline & & P3 & 5 & 10 & 5 & 1 & 0 & 0 & 0.343 \\
\hline & & $\mathrm{P} 4$ & 12.5 & 6 & 5 & 0 & 1 & 0 & 0.330 \\
\hline & & P5 & 17.8 & 12.83 & 5 & -0.47 & -0.88 & 0 & 0.037 \\
\hline & & P6 & 7.2 & 12.83 & 5 & 0.47 & -0.88 & 0 & 0.173 \\
\hline & & P7 & 7.2 & 7.17 & 5 & -0.47 & 0.88 & 0 & 0.243 \\
\hline & & $\mathrm{P} 8$ & 17.8 & 7.17 & 5 & 0.47 & 0.88 & 0 & 0.388 \\
\hline \multirow{3}{*}{$\mathrm{S} 1.2$} & \multirow{3}{*}{0.4} & P9 & 15 & 10 & 3 & 0 & 0 & 1 & 0.1 \\
\hline & & P10 & 10 & 12.5 & 3 & 0 & 0 & 1 & 0.1 \\
\hline & & P11 & 10 & 7.5 & 3 & 0 & 0 & 1 & 0.1 \\
\hline \multirow{3}{*}{$\mathrm{S} 2$} & \multirow{3}{*}{0.2} & $\overline{\mathrm{P} 12}$ & 24 & 12 & 4 & -0.55 & -0.835 & 0 & 0.076 \\
\hline & & $\mathrm{P} 13$ & 24 & 8 & 4 & -0.55 & 0.835 & 0 & 0.305 \\
\hline & & P14 & 22.5 & 10 & 4 & 1 & 0 & 0 & 0.443 \\
\hline
\end{tabular}

$N i$ : Z-component of the vector $\overrightarrow{O P} i \wedge \overrightarrow{n i}$;

$\varphi i=\sqrt{a i^{2}+b i^{2}}=1$ : coefficient of influence of the tool offset radius $\mathrm{R}$ ).

If there are $n$ points on the machined surfaces, a system of $n$ equations is obtained and which can be written in the following matrix form (3):

$$
[a](C)+(-e)=-(\xi),
$$

where $C$ is the vector of correctors.

The matrix $[a]$ is called the incidence matrix because it contains the influence coefficients of correctors on deviations of the points.

This system has no exact solution when the number of points is higher than the number of correctors. The Multiple Linear Regression can then obtain a value of the vector $(C)$ that minimizes the sum of squared deviations. It consists, in his simplest presentation, to multiply the matrix of initial deviations $(\xi i)$ by the pseudo-inverse matrix $\left[a^{*}\right]$ of the incidence matrix using the following equation (4):

$$
(C)=\left[a^{*}\right] \cdot(\xi)
$$

with $\left[a^{*}\right]=\left([a]^{T} \cdot[a]\right)^{-1} \cdot[a]^{T}$

The matrix $\left[a^{*}\right]$ is called steering matrix.

The incidence matrix of the correctors on points expressed in the programming reference frame is given in Table 2 in the red frame. This matrix is calculated using equation (2). Each line of the matrix is obtained thanks to this equation and represents the influence of each corrector $L, R, T x, T y$ and $R z$ on point $P i$.

\section{Consideration of the relative accuracies expressed by tolerances}

The elliptical pocket and the notch have different tolerances. The calculation of corrections by the matrix $\left[a^{*}\right]$ is unfavorable to the surface $\mathrm{S} 2$ witch has the smallest tolerance (see the result of the case \#1 in Tab. 5). So the incidence matrix of Table 3 must be standardizing by
Table 2. Incidence matrix calculated in the programming reference frame.

\begin{tabular}{ccccccc}
\hline Surface & Point & $L$ & $R$ & $T x$ & $T y$ & $R z$ \\
\hline \multirow{5}{*}{ S1.1 } & P1 & 0 & 1 & -1 & 0 & 10 \\
& P2 & 0 & 1 & 0 & -1 & -12.5 \\
& P3 & 0 & 1 & 1 & 0 & -10 \\
& P4 & 0 & 1 & 0 & 1 & 12.5 \\
& P5 & 0 & 1 & -0.47 & -0.88 & -9.63 \\
& P6 & 0 & 1 & 0.47 & -0.88 & -12.37 \\
& P7 & 0 & 1 & -0.47 & 0.88 & 9.7 \\
& P8 & 0 & 1 & 0.47 & 0.88 & 12.3 \\
\hline \multirow{5}{*}{ S1.2 } & P9 & 1 & 0 & 0 & 0 & 0 \\
& P10 & 1 & 0 & 0 & 0 & 0 \\
& P11 & 1 & 0 & 0 & 0 & 0 \\
\hline \multirow{5}{*}{ S2 } & P12 & 0 & 1 & -0.55 & -0.835 & -13.44 \\
& P13 & 0 & 1 & -0.55 & 0.835 & 24.44 \\
& P14 & 0 & 1 & 1 & 0 & -10 \\
\hline
\end{tabular}

Table 3. Standardized incidence matrix.

\begin{tabular}{ccccccc}
\hline Surface & Point & $L$ & $R$ & $T x$ & $T y$ & $R z$ \\
\hline \multirow{5}{*}{ S1.1 } & P1 & 0 & 2.5 & -2.5 & 0 & 25 \\
& P2 & 0 & 2.5 & 0 & -2.5 & -31.25 \\
& P3 & 0 & 2.5 & 5 & 0 & -25 \\
& P4 & 0 & 2.5 & 0 & 2.5 & 31.25 \\
& P5 & 0 & 2.5 & -1.175 & -2.2 & -24.08 \\
& P6 & 0 & 2.5 & 1.175 & -2.2 & -30.91 \\
& P7 & 0 & 2.5 & -1.175 & 2.2 & 24.26 \\
& P8 & 0 & 2.5 & 1.175 & 2.2 & 30.73 \\
\hline \multirow{5}{*}{ S1.2 } & P9 & 2.5 & 0 & 0 & 0 & 0 \\
& P10 & 2.5 & 0 & 0 & 0 & 0 \\
& P11 & 2.5 & 0 & 0 & 0 & 0 \\
\hline \multirow{5}{*}{ S2 } & P12 & 0 & 5 & -2.75 & -4.175 & -67.2 \\
& P13 & 0 & 5 & -2.75 & 4.175 & 122.2 \\
& P14 & 0 & 5 & 5 & 0 & -50 \\
\hline
\end{tabular}

the tolerances of the surfaces. This leads to increase the coefficients of the surface S2 with regard to them of the surface S1. The initial deviations must be also divided by the tolerances to perform the calculations according to 
Table 4. Standardized and balanced incidence matrix.

\begin{tabular}{cccccccc}
\hline Surface & Point & $\begin{array}{c}\text { Repetition number } \\
\text { of the point }\end{array}$ & $L$ & $R$ & $T x$ & $T y$ & $R z$ \\
\hline \multirow{6}{*}{ S1.1 } & P1 & 3 times & 0 & 2.5 & -2.5 & 0 & 25 \\
& P2 & 3 times & 0 & 2.5 & 0 & -2.5 & -31.25 \\
& P3 & 3 times & 0 & 2.5 & 5 & 0 & -25 \\
& P4 & 3 times & 0 & 2.5 & 0 & 2.5 & 31.25 \\
& P5 & 3 times & 0 & 2.5 & -1.175 & -2.2 & -24.08 \\
& P6 & 3 times & 0 & 2.5 & 1.175 & -2.2 & -30.91 \\
& P7 & 3 times & 0 & 2.5 & -1.175 & 2.2 & 24.26 \\
& P8 & 3 times & 0 & 2.5 & 1.175 & 2.2 & 30.73 \\
\hline \multirow{5}{*}{ S1.2 } & P9 & 8 times & 2.5 & 0 & 0 & 0 & 0 \\
& P10 & 8 times & 2.5 & 0 & 0 & 0 & 0 \\
& P11 & 8 times & 2.5 & 0 & 0 & 0 & 0 \\
\hline \multirow{5}{*}{ S2 } & P12 & P13 times & 0 & 5 & -2.75 & -4.175 & -67.2 \\
& P14 & 8 times & 0 & 5 & -2.75 & 4.175 & 122.2 \\
& 8 times & 0 & 5 & 5 & 0 & -50 \\
\hline
\end{tabular}

equation (6):

$$
a i j^{\prime}=\frac{a i j}{t} \quad \text { and } \quad \xi i^{\prime}=\frac{\xi i}{t}
$$

$t$ : tolerance on surface;

aij: incidence of corrector $i$ on the point $j$;

$a i j^{\prime}$ : incidence standardized by tolerances.

Table 3 shows the incidence matrix standardized by tolerances (framed in red).

\section{Consideration of the number of points of surfaces in the normalized matrix}

As it is showed (Tab. 5), the correction obtained by the standardized incidence matrix promotes surfaces machined by the tool with the highest number of points. One solution to remedy this disadvantage is to choose the same number of points on the surfaces affected by the same corrector but it is not always possible in practice. A more general alternative is proposed here. It consists to artificially balance the number of points between surfaces. For this, the same number of points is artificially given back to each of the surfaces. This number of points is corresponding to the least common multiple (LCM) of the numbers of points of surfaces. This balancing of the number of points can give exactly the same weight to each surface.

The surface $\mathrm{S} 1.1$ has eight points $(n 1=8)$, the surface S1.2 three $(n 2=3)$ and the surface S2 three $(n 3=3)$. Their LCM is equal to 24.

The new incidence matrix standardized and balanced has a dimension greater than initially (72 rows and 5 columns instead of 14 rows and 5 columns). This matrix is obtained by duplicating the number of times of measurement points on each surface to reach their LCM of 24. The points on the surface S1.1 are repeated three times and points of surfaces S1.2 and S2 are each of them repeated eight times. Table 4 specifies the number of times (see green frame in the table) that each line is repeated in the incidence matrix that is called standardized and balanced incidence matrix (framed in red in the table).

\section{Comparative results and discussions}

This section compares the results of correction provided by the steering matrices obtained by pseudo-inversion of the incidences matrix, judged by the inertia parameter. An adjustment simulation of the toric milling cutter from the initial deviations $(\xi i)$ of the points given in Table 1 is done. The results are presented in Table 5 , giving the values of corrections to be made to compensate for deviations, as well as gains on the inertia after these corrections.

These results show the gain on inertia that provides the standardization and balancing compared to the raw incidence matrix (case $\# 1$ ). In the case $\# 1$, the gain on inertia is less important on the surface S2 because it have a number of points less than the surface S1.1. In the case \#2 and more so in the case \#3 the gain is improved on the surface S2 without much loss on surface S1.1. Surface S1.1 is logically disadvantaged by balancing. But anyway, this surface still within its tolerance. In all three cases, the gain on surface S1.2 is the same. This is explained by the fact that it depends on one corrector; the tool length offset $L$ of the cutting tool which has no influence on the other surfaces. Standardization and balancing thus have no influence on this corrector.

However, the balancing has the disadvantage to grow the incidence matrix. With LCM of three surfaces equal to 24 , the incidence matrix balanced has 72 lines. Generally, if a tool machines $n$ surfaces with LCM equal to $m$, then this matrix has a number of lines $n \times m$. This disadvantage should nevertheless not be a problem from a point of view of computing time with today's computers. 
Table 5. Comparative study of three incidence matrices.

\begin{tabular}{|c|c|c|c|c|c|c|}
\hline $\begin{array}{l}\text { Incidence } \\
\text { matrix }\end{array}$ & Corrector & Correction & Surface & $\begin{array}{c}\text { Inertia before } \\
\text { correction }\end{array}$ & $\begin{array}{c}\text { Inertia after } \\
\text { correction }\end{array}$ & $\begin{array}{l}\text { Gain on } \\
\text { the inertia }\end{array}$ \\
\hline Case \#1. & Length & -0.1000 & \multirow{2}{*}{ S1.1 } & \multirow{2}{*}{0.2453} & \multirow{2}{*}{0.0194} & \multirow{2}{*}{$92.1 \%$} \\
\hline Incidence matrix & Radius & -0.2290 & & & & \\
\hline without & $T x$ & -0.1558 & \multirow[b]{2}{*}{$\mathrm{S} 1.2$} & \multirow{2}{*}{0.1000} & \multirow{2}{*}{0} & \multirow[b]{2}{*}{$100 \%$} \\
\hline normalization and & $T y$ & -0.1052 & & & & \\
\hline without rebalancing & $R z$ & -0.0012 & S2 & 0.3136 & 0.0524 & $83.3 \%$ \\
\hline Case \#2. & Length & -0.1000 & \multirow[t]{2}{*}{ S1.1 } & \multirow{2}{*}{0.2453} & \multirow{2}{*}{0.0432} & \multirow{2}{*}{$82.4 \%$} \\
\hline Incidence & Radius & -0.2519 & & & & \\
\hline matrix & $T x$ & -0.1651 & \multirow{2}{*}{$\mathrm{S} 1.2$} & \multirow{2}{*}{0.1000} & \multirow{2}{*}{0} & \multirow{2}{*}{$100 \%$} \\
\hline standardized & $T y$ & -0.1070 & & & & \\
\hline by tolerances & $R z$ & -0.0012 & $\mathrm{~S} 2$ & 0.3136 & 0.0288 & $90.8 \%$ \\
\hline Case \#3. & Length & -0.1000 & \multirow[t]{2}{*}{ S1.1 } & \multirow{2}{*}{0.2453} & \multirow{2}{*}{0.0576} & \multirow{2}{*}{$76.5 \%$} \\
\hline Incidence & Radius & -0.2660 & & & & \\
\hline matrix & $T x$ & -0.1705 & \multirow{2}{*}{$\mathrm{S} 1.2$} & \multirow{2}{*}{0.1000} & \multirow{2}{*}{0} & \multirow{2}{*}{$100 \%$} \\
\hline standardized & $T y$ & -0.1076 & & & & \\
\hline and balanced & $R z$ & -0.0012 & $\mathrm{~S} 2$ & 0.3136 & 0.0143 & $95.5 \%$ \\
\hline
\end{tabular}

Table A.1. Graphics and values of deviations of the points before and after correction.

\begin{tabular}{|c|c|c|c|c|c|c|c|c|c|c|c|c|c|c|c|}
\hline Point & $\begin{array}{c}\text { Initial } \\
\text { deviation }\end{array}$ & & & & $\begin{array}{l}\text { Residues } \\
\text { deviation } \\
\text { Case \#1 }\end{array}$ & & & & $\begin{array}{l}\text { Residues } \\
\text { deviation } \\
\text { Case \#2 }\end{array}$ & & & $\begin{array}{l}\text { Residues } \\
\text { deviation } \\
\text { Case \#3 }\end{array}$ & & & \\
\hline $\mathrm{P} 1$ & 0.077 & 0.5 & & $\begin{array}{ll}1.0 & 0.5\end{array}$ & -0.009 & -0.5 & 0 & 0.5 & -0.022 & 0.5 & 0.5 & -0.031 & -0.5 & 0 & 0.5 \\
\hline $\mathrm{P} 2$ & 0.090 & & & 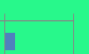 & -0.018 & & 1 & & -0.04 & 1 & & -0.053 & & 11 & \\
\hline P3 & 0.343 & & 2 & 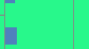 & -0.029 & & 2 & & -0.062 & 21 & & -0.081 & & 21 & \\
\hline $\mathrm{P} 4$ & 0.330 & & 3 & 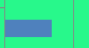 & -0.02 & & 3 & & -0.044 & 3 & & -0.059 & & 3 & \\
\hline P5 & 0.037 & & 4 & 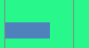 & -0.014 & & 41 & & -0.031 & 4 & & -0.042 & & 4 & \\
\hline P6 & 0.173 & & 5 & & -0.021 & & 5 & & -0.047 & 51 & & -0.063 & & 5 & \\
\hline $\mathrm{P} 7$ & 0.243 & & 6 & 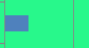 & -0.017 & & 01 & & -0.037 & 6 & & -0.05 & & $6=$ & \\
\hline P8 & 0.388 & & 7 & $=$ & -0.022 & & 7 & & -0.051 & $7 !$ & & -0.068 & & 7 & \\
\hline P9 & 0.1 & & 8 & 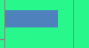 & 0 & & 8 & & 0 & $8 !$ & & 0 & & 8 & \\
\hline P10 & 0.1 & & 9 & 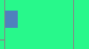 & 0 & & 9 & & 0 & 9 & & 0 & & y & \\
\hline $\mathrm{P} 11$ & 0.1 & & & 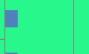 & 0 & & 10 & & 0 & 10 & & 0 & & 10 & \\
\hline $\mathrm{P} 12$ & 0.076 & & 1 & 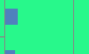 & 0.037 & & 11 & & 0.021 & 11 & & 0.01 & & 11 & \\
\hline $\mathrm{P} 13$ & 0.305 & & 3 & & 0.043 & & \begin{tabular}{l|l}
12 & $\mid$ \\
13
\end{tabular} & & 0.025 & $\begin{array}{l}12 \\
13\end{array}$ & & 0.012 & & $\begin{array}{l}12 \\
13\end{array}$ & \\
\hline $\mathrm{P} 14$ & 0.443 & & & ( & 0.071 & & 14 & & 0.038 & 14 & & 0.019 & & 14 & \\
\hline
\end{tabular}

\section{Conclusion}

The principle of Total inertial steering approach is presented here for the case of a single tool machining surfaces with different tolerances. This approach for setting machining has the advantage to be generally to any type of surface and able to take into account any type of correction. It has been shown that to be effective towards conformity parts, it should take into account the tolerances of the different surfaces by standardizing incidence matrix by tolerances. Finally balancing surfaces in the incidence matrix makes it possible not having to worry about the number of points to be probed on each surface. They can be very different; the results are the same. The gain on inertia of the proposed approach has been shown from a simple example. In the application example given in the article, the gain move of $83.3 \%$ on inertia in the case \#1 on the surface S2 having the smallest tolerance and the smallest number of points, to a gain of $95.5 \%$ in case \#3. However there remains work to be done to propose the number of points and their positions on the surface to future users of this method.

\section{Appendix: Deviations of the points before and after correction}

Initial deviations and final deviations calculated with each incidence matrix studied in this paper are report in Table A.1. The position of these deviations before and after correction for each case of matrix is graphically showed. 


\section{References}

1. D. Denimal, M. Pillet, A. Sergent, "The adjustment and monitoring of freeform surfaces using inertial tolerancing", Quality Assurance, January- March 2011

2. M. Pillet, Améliorer la productivité, déploiement industriel du tolérancement inertiel, EYROLLES, Editions d'Organisation, 2010, ISBN: 978-2-212-54754-2

3. S. Tichadou, O. Legoff, J.-Y. Hascoët, Quantification of machining and fixture dispersions for Geometrical manufacturing simulation, CIRP-CAT 2007

4. A. Sergent, H. Bui-Minh, H. Favrelière, D. Duret, S Samper, F. Villeneuve, Identification of machining defects by small displacement torsor and form parameterization method, Paper Proposed at the IDMME-Virtual Concept 2010 International conference, Bordeaux, France, 2010

5. L. Zilong, E. Del Castillo, Setup adjustment under unknown process parameters and fixed cost, Department of Industrial and Manufacturing Engineering, The Pennsylvania State University, Journal of statistical planning and inference, 2004

6. Del Castillo, E. Rong, Bianca P.M. Colosimo, An unifying view of some process adjustment methods, Department of Industry \& \&anufacturing Engineering, The Pennsylvania State University, University Park, PA 16802, Dipartimento di Meccanica, Politecnico di Milano, 2002

7. P. Rong, Statistical process adjustment methods for quality control in short-run manufacturing, Ph.D. Thesis in Industrial Engineering, Department of Industrial and Manufacturing Engineering, The Pennsylvania State University, 2002
8. F.E. Grubbs, An optimum procedure for setting machines or adjusting processes, J. Quality Technol. 15, 186-189 (1954)

9. P. Martin, P. Schneider, J.-Y. Dantan, Optimal adjustment of a machine tool for improving the geometrical quality of machined part, Int. J. Adv. Manuf. Technol. 26, 559-564 (2005)

10. E. Pairel, E. Goldschmidt, B. Vayre, P.A. Adragna, M. Pillet, COPILOT PRO ${ }^{\circledR}$ : A full method for the steering of the machining, Int. J. Metrol. Qual. Eng. 2, 39-44 (2011)

11. E. Pairel, E. Goldschmidt, B. Vayre, B. Abdelhakim, M. Pillet, Process plans and manufacturing dimensions for the steering of machining: The Copilot-Pro ${ }^{\circledR}$ methodology, 12th CIRP Conference on Computer Aided Tolerancing, Huddersfield, UK, April 2012

12. E. Goldschmidt, Gamme et cotation pour le réglage des machines-outils de décolletage, Ph.D. thesis, Université de Savoie, Annecy, France, 2009

13. M. Pillet, É. Pairel, Determination of an adjusting rule in the case of multi-criteria inertial piloting, Int. J. Metrol. Qual. Eng. 2, 51-59 (2011)

14. M. Pillet, É. Pairel, J.L. Maire, B. Abdelhakim, Différentes stratégies de filtrage en pilotage inertiel multicritères, CPI'2011, 2011

15. P. Bourdet, A. Clement, A study of optimal - criteria identification based on the small - displacement screw model, Ann. CIRP 37/1/1988, 1988 